\title{
Téoros
}

Revue de recherche en tourisme

\section{Éléments pour une économie du tourisme social}

\section{Paul Bodson, Jean Stafford et Richard Nicol}

Volume 1, numéro 3, 3e trimestre 1982

Les enjeux du tourisme social

URI : https://id.erudit.org/iderudit/1080848ar

DOI : https://doi.org/10.7202/1080848ar

Aller au sommaire du numéro

Éditeur(s)

Université du Québec à Montréal

ISSN

0712-8657 (imprimé)

1923-2705 (numérique)

Découvrir la revue

Citer cet article

Bodson, P., Stafford, J. \& Nicol, R. (1982). Éléments pour une économie du tourisme social. Téoros, 1(3), 22-25. https://doi.org/10.7202/1080848ar d'utilisation que vous pouvez consulter en ligne.

https://apropos.erudit.org/fr/usagers/politique-dutilisation/ 


\title{
Éléments pour une économie du tourisme social
}

\author{
Par Paul Bodson et Jean Stafford
}

\section{Derrière les études, des enjeux d'envergure!}

Par Richard Nicol, Groupe de ressources en tourisme social

Depuis plus de deux ans, une "animation fertile" a conduit les associations nationales préoc cupées de tourisme social à investir la scène publique. Elles ont développé un discours éla boré (qui n'est pas toujours suivi d'une pratique correspondante) et multiplié des initiatives d'affirmation aux plans social et économique. L'heure est cependant venue de démontrer, chiffres et paramètres à l'appui, notre véritable pouvoir économique et social: quelles sont donc les réalités qui s'agitent derrière les grandes idées? Si les associations nationales ont crié haut et fort leur rentabilité, elles font maintenant face à leurs détracteurs qui exigent des preuves tangibles: les marchands de rêves et leurs alliés ne s'en laisseront pas imposer aussi facilement! Adoptons donc leurs propres moyens de propagande pour justifier concrètement notre propre valeur sur le terrain.

Pour ce faire, des études et des recherches complexes apparaissent tout à fait essentielles, afin:

a) de prouver la rentabilité sociale et culturelle de nos expériences et de démasquer les seules préoccupations réelles de l'industrie touristique, la recherche du profit et l'accumulation du capital;

b) d'établir la rentabilité sociale et culturelle de nos expériences et de démasquer les seùles préoccupations réelles de l'industrie touristique, la recherche du profit et l'accumulation du capital:

c) de justifier clairement le rendement des investissements gouvernementaux dans le secteur du tourisme social et d'affirmer ainsi la valeur de notre apport au développement communautaire.

Le contexte de crise, qui perdure, ne pardonnera aucune erreur stratégique: si l'on veut éviter que le ballon idéologique se dégonfle tout bêtement, il est essentiel de passer aux actes probants. Cependant, cette grande entreprise de démonstration comporte des risques tout aussi stimulants qu'inquiétants. En effet, il n'est pas évident que l'économie du tourisme social puisse rivaliser avantageusement avec les "statistiques" de l'industrie touristique payées à même nos taxes. De plus, si l'on fouille attentivement les exigences de la rentabilité sociale et culturelle, il n'est pas sûr que les expériences québécoises franchis: sent les sommets atteints par nos homologues français; au contraire, il s'agit d'un terrain piégé.

Par contre, si ces études permettent d'aller de l'avant plus encore et de réajuster des tirs imprécis, nul doute que nous en ressortirons grandis en crédibilité, en innovation et en maturité.

Au moment même oủ less associations doivent amorcer une coordination de leurs efforts et développer une solidarité exemplaire, elles font face au repliement sur soi, à l'apathie et à l'indifférence. Elles devront secouer le moral de leurs troupes et injecter des sommes dans un programme intégré d'études et de recherches. Celà se justifie-t-il en pleine récession? Trouverat-on la vitalité requise par une telle démarche collective? C'est maintenant, et non plus tard, que nous pourrons jauger la force collective des associations et du milieu préoccupés par le développement du tourisme social. Chose certaine, si les généreuses politiques ne se concrétisent pas, le tourisme social risque de sombrer tout bonnement dans la solitude historique plutôt que d'exercer un leadership emballant dans la mise en place d'un mouvement national de défense du droit aux vacances. Quel jugement portera-t-on dans cinq ans, sur les attitudes et les démarches qui seront adoptées bientôt? C'est un pensez-y bien car nous devront affronter la critique sociale à un moment ou à un autre, inévitablement.
Richard Nicol du G.R.T.S. prône la nécessité de cerner les véritables dimensions économiques du tourisme social. C'est un débat qui a cours depuis quelque temps dans le milieu du tou. risme social.

Les économistes Paul Bodson et Jean Stafford apportent leur contribution à ce débat (N.D.L.R.)

Une économie du tourisme social est-elle possible? C'est à cette question que nous tenterons de répondre dans cet article. II s'agit pour nous d'élaborer certains principes, de formuler des concepts et des méthodes permettant de mieux cerner les dimensions économiques du tourisme social. Ce travail est une première réflexion et les éléments avancées ici devront être discutés et approfondis davantage.

Nous confronterons tout d'abord les caractéristiques du tourisme commercial et du tourisme social. Globalement, le tourisme commercial recherche en priorité, le profit. Par contre, le tourisme social se distingue par une conception normative du rôle du tourisme dans la société.

Le tourisme social s'intéresse surtout à la rentabilité sociale du tourisme; le tourisme commercial s'intéresse au profit. L'un et l'autre constituent des formes différentes de percevoir et de vivre une même réalité. Ces formes devront donc être étudiées selon des approches distinctes. II faudra adapter les instruments d'analyse traditionnelle et si nécessaire, forger de nouveaux instruments pour approcher le phenomène du tourisme social.

L'économie du tourisme commercial Lorsqu'il s'intéresse au tourisme, un individu ou un organisme privé mû par la recherche du profit, concoit d'abord les services qu'il offre à la popularion comme un moyen d'arriver à ses fins. Pour être efficace dans son projet, le spécialiste en tourisme commercial se préoccupera des attentes de la population, écoutera, en d'autres termes, la demande que lui manifeste la population. Sans souci explicite de la valeur sociale de la demande, il cherchera à y répondre de manière à en tirer le maximum de profit. 


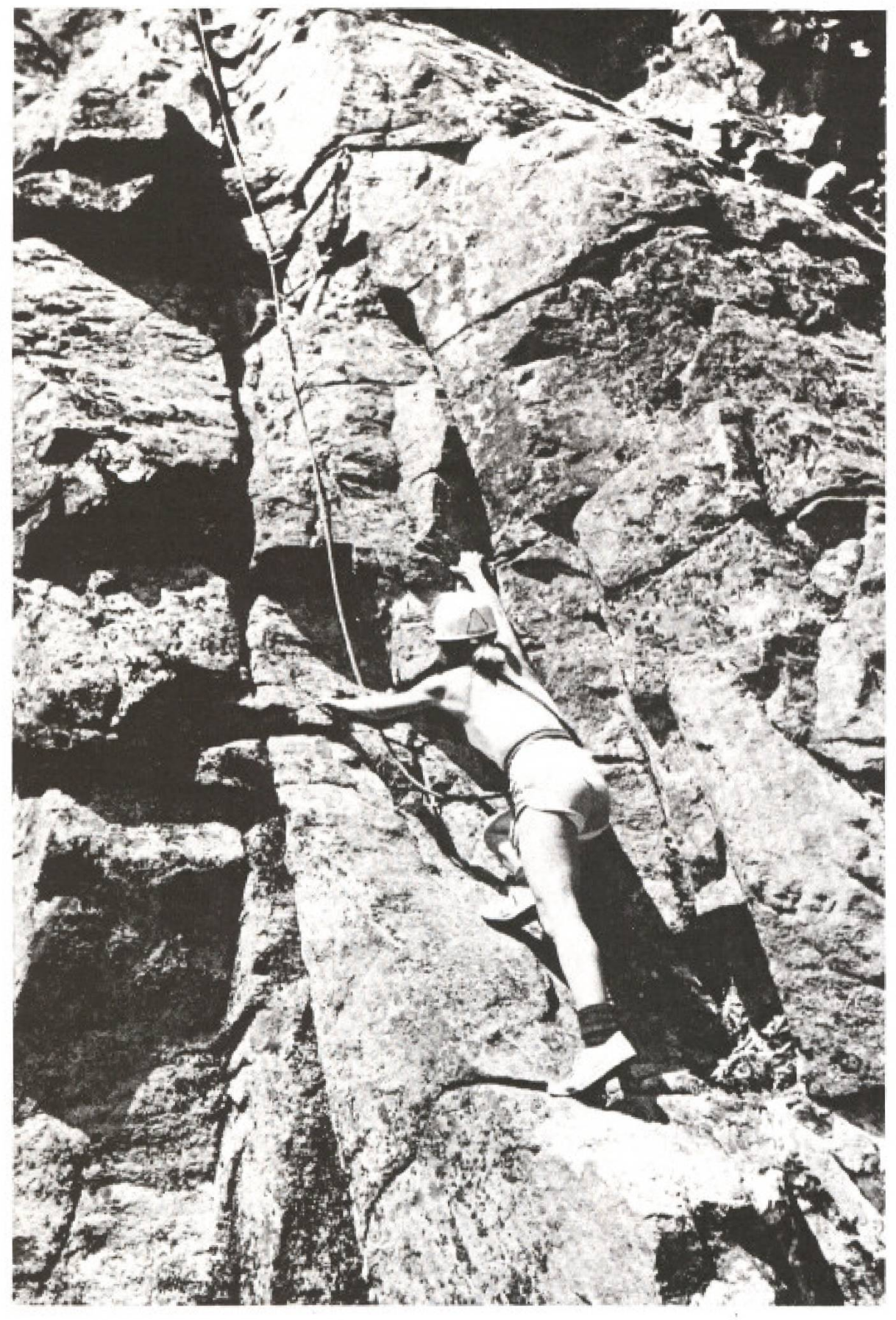

Éventuellement, s'il est possible de manipuler les goûts de la population, il ira jusqu'à orienter les activités touristiques de manière à maximiser son profit.

Peu de spécialistes en tourisme se reconnaittraient dans cette image. De fait, la seule nécessité de conformer l'offre de tourisme à la demande de la population, jointe à l'impossibilité de manipuler complètement les goûts de la population, aboutissent à des réalisations de la part du secteur commercial répondant partiellement aux valeurs poursuivies dans une société. De plus, la froideur du comportement axé sur le seul profit est rarement pure. En agissant, toute personne poursuit la recherche d'une satisfaction plus globale qui relève de l'ensemble des valeurs qui l'animent, et qui, sou- vent, peuvent rejoindre les valeurs promues dans le développement d'une société.

La poursuite du profit, comme objectif prioritaire, conférant le statut de "moven" aux services offerts à la population, conditionne néanmoins la structure du tourisme commercial et demande à ce titre d'ettre explicitée.

Devant les perspectives d'un projet touristique commercial, se pose tout d'abord la question de la rentabilité. Comme le mot le suggère, est rentable une activité susceptible de procurer une rente, c'est-à-dire finale coüt impliqué dans l'opération, en raison des conditions favorables dans lesquelles se déroule l'activité. La rente peut être nulle. lement une marge de bénéfices dépassant
Une telle situation provoquera rapidement une réaction des personnes poussées par la recherche du profit - c'est vraisemblablement le cas pour de nombreux bailleurs de fonds. C'est pourquoi, par elle-mème, une activité à rente nulle est généralement vouée à la disparition. Les détenteurs de fonds préféreront s'orienter vers des activités aptes à générer le maximum de profit. Dans la mesure où elle est soumise à cette contrainte, l'activité touristique commerciale cherchera dès lors à s'assurer les conditions optimales de rentabilité, c'est-à-dire dans ce contexte, les meilleures conditions de profit.

Lors de l'évaluation d'un projet, deux types de considération entrent en jeu. La première interrogation porte sur la rentabilité globale du projet, la seconde sur le prélèvement du maximum de profit si on suppose données l'infrastructure et I'association équipement personnel.

Dans une perspective à long terme, on s'interrogera sur le profit que peut générer un projet si on le considère dans l'ensemble du cycle de son existence. II s'agit, dans cette optique, d'evaluer les recettes et les coûts à chaque période du cycle de vie du projet, d'en obtenir une image d'ensemble et de la confronter avec d'autres projets éventuellement réalisables. Est finalement retenu le projet qui offre le profit d'ensemble le plus élevé. Ce type d'appréciaation globale se fait souvent à l'aide du calcul d'actualisation. La logique du calcul d'actualisation consiste à ramener au présent les coûts et les recettes générés à chaque période de la vie d'un projet à l'aide d'un taux d'actualisation. Le taux d'actualisation permet de calculer les sommes dont il faudrait disposer maintenant pour obtenir, par placement au taux d'inté rêt en vigueur, les sommes (en termes de recetes et de coûts) générés à chaque période du cycle du projet.

A court terme, la logique du profit poussera le tourisme commercial à offrir des services aussi longtemps qu'ils sont générateurs de recettes superieures aux coûts; mais le tourisme commercial se gardera bien d'augmenter ses services si le service supplémentaire provoque un coût supérieur à la recette. Un tel service diminuerait en effet le volume global du profit. La logique du profit pousse donc le tourisme commercial à maintenir ses services en deça du volume de services qu'il est techniquement possible d'offrir à la population.

\section{Tourisme commercial ou} tourisme social

L'utilisation que l'on fait dans notre système de connaissance, de l'économie du projet et du discours social ne sont pas neutres; elles correspondent à une vision réductrice de la vie sociale. L'homme intéressé par le profit semble vivre à l'écart de l'homme sociologique; c'est pourtant la mème personne quil vit et agit à l'intérieur d'une société donnée. 
On oppose souvent l'économie du profit et le social des quatre facons suivantes ${ }^{16 !}$ :

$\begin{array}{lr}\text { l'économie du profit } & \text { le social } \\ \text { les objets } & \text { les personnes } \\ \text { l'individu } & \text { les groupes } \\ \text { la demande solvable } & \text { les besoins } \\ \text { les moyens } & \text { les fins }\end{array}$

L'économie du profit a pour principale tâche de produire des objets alors que le social s'occupe des personnes. L'économie du profit privilégie les valeurs individuelles, le social insiste sur les dimensions collectives des phénomènes. L'économie du profit correspond a une demande solvable, le social se préoccupe de besoins fondamentaux. Enfin l'économie du profit est le monde des moyens et le social celui des fins; le domaine économique a pour principale tãche de mesurer et de déterminer ce qui est rentable ou non; le social sert plutớt à définir des objectifs apparemment plus vagues sinon irréalistes.

Ces quatre oppositions sont souvent utilisees pour remettre "le tourisme social à sa place" résiduel de l'ensemble du tourisme. Présenté de cette facon, le tourisme social n'est plus qu'un problème d'assistance, un substitut coùteux au tourisme "rentable". Le tourisme social est ainsi carrément rejeté de la sphère économique. En réalité, les catégories économiques à un moment donné sont le produit d'une société à un moment donné; elles sont imbriquées dans le social; l'économique et le social sont en relation dia lectique et dépendent d'une histoire commune.

\section{Le tourisme social produit \\ des biens collectifs}

La meilleure facon de dépasser les oppositions décrites plus haut est de définir le tourisme social comme producteur de biens collectifs. La production de biens collectifs est une fonction économique importante: elle est caractérisếe, en général, par la recherche d'un optimum social; elle est donc habituellement sans but lucratif.

II existe differents types de biens collectifs dans une société. On les distingue par leur niveau d'accessibilité et leur niveau d'exclusion. II existe tout d'abord des biens collectifs qui admettent une utilisation, sans des. truction par l'utilisateur et sans exclusion de qui que ce soit Ichaleur du soleili I'air qu'on respire, la rue où l'on marchel. Le deuxième type de biens collectifs suppose une sim. ple exclusion: si jutilise un canot dans une base de plein air, j'empêche quelqu'un d'autre de l'utiliser au même moment. Le troisième et dernier type de biens collectifs comprend une double exclusion: si un camp de vacances est réservé à une catégorie sociale particulière (assistés sociaux, etc...), elle exclut d'autres catégories sociales (première exclusion); la deuxième exclusion correspond au deuxième type de biens collec- tifs lexclusivité d'un équipement à un moment donné pour un temps donnél). Le tourisme social produit surtout des biens collectifs du $2 e$ et du $3 e$ type.

\section{Les critères permettant de définir le tourisme social comme producteur de biens collectifs}

Le premier de ces critères précise l'accessibilité des biens collectifs produits par le tourime social à des catégories sociales bien définies. Selon le Bureau international du tourisme socialai les biens collectifs produits par le tourisme social sont surtout réservés aux couches sociales les plus démunies de notre société. C'est un objectif social qui justifie la production de biens collectifs.

Le deuxième critère va porter sur les fonctions sociales que peut remplir le tourisme social; ces fonctions sont une autre façon de cerner le concept de biens collectifs. L'addition de ces fonctions permet de mesurer la rentabilité de ces biens collectifs. Ces fonctions peuvent être:

- le degré d'insertion des organismes sans but lucratif au niveau local, régional et mème national:

- la participation de ces organismes à l'aménagement local et régional;

- la protection du patrimoine physique, his torique et culturel assurée par les organismes du tourisme social;

- la formation permanente des individus et des groupes: cette formation découle des critères cités plus haut et de l"apprentissage effectué par tous les usagers du tourisme social à travers les valeurs et les modèles de comportement proposés par les organismes du tourisme social. Cette liste n'est pas exhaustive et d'autres critères pourraient s'ajouter à ceux déjà mentionnés.

Le troisième critère concerne la productivité des organismes du tourisme social. II s'agit d'établir la performance des orgnismes concernés en fonction des objectifs visés. L'éta blissement de cette performance suppose la formulation de trois types d'indicateurs:

\section{- indicateurs d'objectifs \\ - indicateurs de movens \\ - indicateurs des résultats}

L'adéquation entre les indicateurs d'objectifs à atteindre et les indicateurs des résul. tats obtenus à une période $\mathrm{t}_{\mathrm{n}}$ peut être, en soi, une bonne mesure de la performance d'un organisme sans but lucratif.

Le quatrième critère est sensiblement plus vague; il s'agit de répondre à la question: jusqu'à quel point les organismes du tou. risme social répondent-ils aux besoins de la collectivite globale? Ces besoins sont sou- vent difficile à circonscrire parce qu'ills sont nombreux et souvent contradictoires. L'analyse des grandes tendances sociales dans une société donnée pourrait nous aider à cerner ces besoins; par exemple deux grandes tendances sociales au Canada, ont été isolées par D.W. Henderson ${ }^{10}$; ces tendances sont:

1. la recherche d'une plus grande équité sociale et economique;

2. un intérèt accru envers des objectifs socio-économiques à tendance sociale.

A contrario, on pourrait définir les besoins de la société à partir des coûts du laissezfaire. Par exemple on pourrait émettre l'hypothèse que le fait de ne pas prendre des vacances annuelles est un facteur de stress producteur de maladies mentales et physiques. Les coûts de l'inaction de la part des pouvoirs publics pourraient être mesurés en terme des coûts des services médicaux et sociaux encourus à cause de ce manque dans la politique sociale. Des recherches récentes ${ }^{|1|}$ portant sur le coüt des accidents de la circulation, le coût du tabagisme et des maladies permettent de croire que cette comptabilité est possible.

Un cinquième critère précise l'optimalité des biens collectifs en termes d'intervention des pouvoirs publics. Les pouvoirs publics peuvent produire eux-mèmes les biens collectifs jugés importants pour la société. Ils peuvent aussi intervenir de manière indirecte en subventionnant des organismes intéressés par le profit ou des organismes sans but lucratif. La subvention aura pour but $s^{\prime}$ assurer le volume, la qualité, l'accessibilité des services que les pouvoirs publics souhaitent voir mis à la disposition de la population, en particulier des couches de revenus plus défavorisées.

\section{Les méthodes d'analyse des biens collectifs produits par le tourisme social}

II s'agit ici d'indiquer par quels movens on pourra mesurer les critères énoncés tout-à l'heure. Nous allons reprendre chacun de ces critères qui défiissent le tourisme social comme producteur de biens collectifs et suggérer des méthodes de mesure appropriées.

Le critère d'accessibilité peut être mesuré grăce à une étude des usagers de ces orga. nismes du tourisme social; I'analyse du profil des usagers nous permettra de constater si, ceux-ci, correspondent let jusqu'at quel pointl aux publics-cibles déterminés au départ. Si l'écart est trop grand entre le profil supposé des usagers et le profil reel il fau. dra envisager, soit une redéfinition des objectifs sociaux, soit des programmes visant â atteindre ces objectifs. Les méthodes de recherche sont bien connues et figurent sous la rubrique du marketing social. ${ }^{\text {. }}$ 
Les fonctions sociales des biens collectifs définis par le tourisme social vont être isolées, répertoriées et analysées à partir d'indicateurs sociaux. ${ }^{[\overline{\mid} \mid}$ La formulation de ceux-ci suppose un long travail de conceptualisation et d'interprétation. Une fois mis en place, ceux-çi pourraient donner un éclairage nouveau sur les services rendus à la collectivité par la production de biens collectifs produits par les organismes du tourisme social. Les dimensions habituelles de la méthode avantages/coûts pourraient être revisées de facon à faire "éclater" cette analyse, actuellement trop restrictive, en incluant d'autres dimensions propres à mieux expliquer les avantages et les coûts des biens collectifs étudiés.

Le critère de productivité peut se mesurer de plusieurs facons. Une mesure simple serait par exemple d'établir le taux de remplissage des lits sociaux et de le comparer au taux de remplissage du secteur privé. Une autre facon serait aussi de comparer les subventions obtenues dans l'un ou l'autre des secteurs. ${ }^{17}$ Enfin une méthode plus complexe serait d'élaborer des comptes satellites du tourisme au niveau local et régional: ${ }^{48}$ le compte satellite est une comptabilité semblable à la comptabilité nationale mais appliquée à un domaine précis (par exemple, le tourisme). Les comptes satellites pourraient permettre d'établir un bilan annuel au niveau régional en faisant la comptabilité de l'ensemble des comportements socio-économiques reliés au tourisme.
L'étude des objectifs sociaux que veut se donner une socièté peut se faire par une analyse de sondages portant sur ce theme, L'étude des valeurs sociales peut aussi être réalisée par l'analyse de la place des biens collectifs par rapport à ce qui est dépensé pour les biens privés dans une société donnée. L'analyse comparative des politiques sociales de difftérents pays (par exemple. le chèque vacancest en matière de tourisme social pourrait nous aider à déterminer les valeurs sociales dominantes dans une collectivité.

L'intervention des pouvoirs publics, quelle soit directe ou indirecte, lors de la création de biens collectifs peut être appréciée en termes d'efficacité par rapport aux objectifs poursuivis. Des niveaux différents de services peuvent être atteints selon l'affectation des depenses issues des pouvoirs publics. II $\mathrm{y}$ aura lieu, dans cette perspective, de recourir à des mesures de productivité en termes de dépenses impliquées, tout en tenant compte des finalités poursuivies par les organismes subventionnés. Une subvention accordée à un secteur du tourisme commercial impliquera quasi automatiquement un tribut payé au profit. Une subvention accordée à un secteur sans but lucratif pourra éventuellement aboutir à une efficacité apparemment plus faible parce que ce secteur est soumis à une diversité d'objectifs, l'insertion dans le contexte régional par exemple, en plus de la création de services spécifiques.

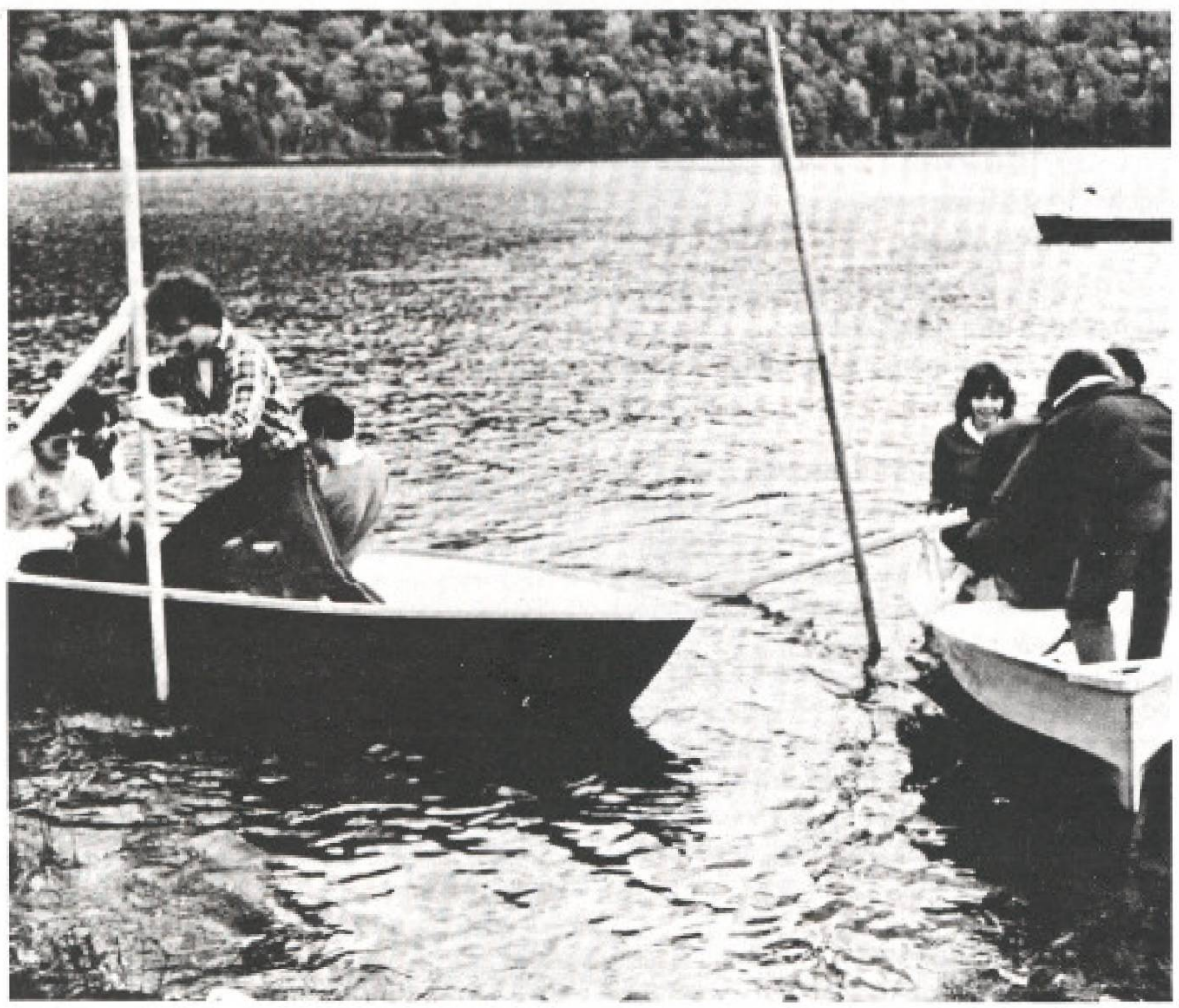

Conclusion

A traves la confrontation entre le tourisme commercial et le tourisme social se dégage une double conclusion.

A côté du tourisme commercial, it s'impose de donner une place spécifique au tourisme social, parce qu'il répond à un objectif propre: la creation d'un certain type de biens collectifs.

II existe un ensemble de méthodes relativement rigoureuses pour apprécier l'efficacité du tourisme social. Ces méthodes sont distinctes des méthodes couramment utilisées dans le tourisme commercial. Le recours à ces dernières pour évaluer le tourisme social provoquerait un biais majeur d'analyse et de compréhension.

Avec William Kapp nous croyons "qu'en incluant is coüts sociaux, les avantages sociaux et la valeur sociale dans le cadre de son analyse, la science économique deviendrait l'économie politique dans un sens plus profond et phus large que celui que les économistes classiques eux-mémes donnaient á ce terme.

\section{Bibliographie}

(11 Voir: GREFFE, X, La politique sociale, P.U.F, Paris, 1975, De. $15 \cdot 20$

(2) Voir DULUDE, N, MOLIN, L, Les infervenanis tounistizures in NADEAU, $R$, ef 혜. Lê tourisme Aspects théoriques et pratiques au Ouebec. SODius, Mansteal. 1982. p. 200.

(3) Voir: HENDERSON DW, Les indicateurs sociaux, Expese analytique et cadre de racherche, Conseil Economipue div Canoda, Ortawa, 1974, op. 73-100

(4) Voir: LE NET, M. Le prix de la vie humaine, La Documen. tation Francaise, Paris, 1978 .

5) Voir les classiques dans ce domaine LINDON, D. Marke. ting politique et social, Dalloz, Paris, 1976; BON, J. LOUPPE. A. Marketing des survices publics: l'étude des besoins de la population, Les deditions dorganisation. Paris. 1980.

16. Pour un sou dhorison woir: BALEA, R.A., Social Indicators. The M.1.T. Pness, Cambridge, 1966;

DELORS, \& Les indicateurs sociaux, Ed Futuribles, Paris, 1971 :

LAND K SPILERMAN, S. Social indicators Modets, FLS 5el Soge Foundation, N.Y., 1975

(7) Voir: - Economie du tourisme social, U.C.E.L. Pars 1980

(18) Voir: - Amenagement de lespace et du temps et developpement du tourisme, Le Documentation Francaisc Paris, 1981 , D $105-106$

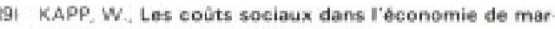
che, Fismmarian, Paris, 1963, p. 334 